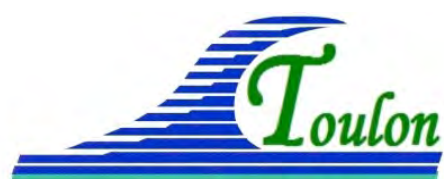

\title{
Etude courantologique de la partie nord du lagon de Wallis et schéma de circulation générale
}

\author{
Thierry HOIBIAN ${ }^{1}$, Michel ALLENBACH ${ }^{1}$, Enelio LIUFAU ${ }^{2}$, Karine BRUNET $^{2}$, \\ Atoloto MALAU ${ }^{2}$, Falalika TUHIMUTU-TAOFIFENUA ${ }^{2}$
}

\author{
1. Université de la Nouvelle-Calédonie, PPME - EA3325, BP R4, \\ 98851 Nouméa Cedex, Nouvelle-Calédonie. \\ thierry.hoibian@univ-nc.nc;michel.allenbach@univ-nc.nc \\ 2. Service Territorial de l'Environnement, BP 294 - Mata'Utu - Uvea, \\ 98600 Wallis et Futuna.senv@mail.wf ; sig.env@mail.wf ; labo.env@mail.wf
}

\section{Résumé :}

De nouvelles mesures courantologiques réalisées sur des stations situées dans la partie nord du lagon de Wallis, confirment schéma de circulation global présenté antérieurement et précisent les influences respectives des paramètres agissant sur les circulations (vents, marées et houles). Les observations effectuées sur des stations de la partie nord du lagon sont présentées. Elles montrent l'écoulement prépondérant des eaux du lagon nord-est vers le lagon nord-ouest et le développement d'une circulation à deux couches: une couche de surface, mobile, avec des courants rapides, influencée par les apports des houles océaniques et le vent mais peu influencée par la marée, une couche de fond, avec des courants moins rapides, davantage influencée par la marée et la morphologie du fond. L’épaisseur de la couche de surface augmente avec la sévérité des conditions météorologiques.

Mots-clés : Courantologie, ADCP, Lagon, Marée, Houle, Iles Wallis, Pacifique Sud-Ouest.

\begin{abstract}
:
New ADCP courantologic measurements from some stations located in the northern part of the Wallis island lagoon, confirm the global circulation pattern described previously and provide details of circulations. Respective influences of parameters affecting the circulation (winds, tides and waves), are specified. Observations show the dominant outflow of the waters of the north-eastern part of the lagoon toward northwestern lagoon and the existence of a two layers circulation: a highly mobile surface layer with fast currents, influenced by oceanic swells inflows and winds with a weak influence of tides, a bottom layer with slower currents, but a stronger influence of tide and control of the bottom morphology. Thickness of the surface layer increases with the severity of meterologic conditions.
\end{abstract}

Keywords: Current, ADCP, Lagoon, Tide, Swells, Wallis islands, South-West Pacific. 


\section{Introduction}

Positionnées par $13^{\circ} 16^{\prime}$ Sud et $176^{\circ} 15^{\prime}$ ' Ouest, les iles Wallis et Futuna sont des Territoires français d'outre-mer situé dans la zone intertropicale du Pacifique sud-ouest. L'île de Wallis est la partie émergée d'un énorme volcan éteint, qui culmine à une altitude de $150 \mathrm{~m}$. L'île principale est longue de $14 \mathrm{~km}$ et large de $7 \mathrm{~km}$. Elle est entourée d'une large barrière de corail, délimitant un lagon d'une largeur de 3 à $5 \mathrm{~km}$, ouvert sur l'océan par quatre passes étroites. Ce site fait l'objet d'une étude océanologique depuis 2005, dans le cadre du suivi de l'impact du réchauffement climatique des Territoires d'outre-mer. Ce dernier volet de l'étude courantologique, dont les premiers résultats ont étés publiés antérieurement, présente les résultats des mesures réalisées à la partie nord du lagon. Grâce à ces nouvelles acquisitions de données, le modèle de circulation global déjà proposé a pu être validé, les modalités des circulations affinées et les forçages définis par CHEVALIER et al., (2015) évalués.

\section{Matériel et méthodes}

Le profileur de courants utilisé pour l'étude courantologique est un ADCP Nortek 600 $\mathrm{kHz}$. Cet instrument mesure les vitesses et directions des courants à différents niveaux de la tranche d'eau, appelés cellules, de trois à six selon la profondeur. Il a été immergé sur différentes sites du lagon nord, pour des sessions d'une durée de un à six mois en moyenne. Le pas d'échantillonnage a été fixé à 30 minutes. Les résultats de quelques stations de courantologie situées dans la partie nord du lagon sont examinés ;

- trois stations de chenal interne ; Hihifo, Nuku Fufulanoa, et passe de Vailala,

- une station de pleine eau dans le lagon nord-ouest ; Vailala nord,

- une station positionnée à proximité de la barrière ouest ; Te Fasi.

La méthodologie d'étude des courants utilisée a été exposée dans deux communications précédentes (HOIBIAN et al., 2012 ; HOIBIAN et al., 2014). Différents niveaux d'analyse sont appliqués. 1 - une analyse globale pour l'ensemble de la session, pour chaque cellule, qui donne les grandes tendances de la circulation hydraulique pour un site donnée, 2 - une étude détaillée, réalisée pour une période particulière d’une durée de 5 à 6 jours qui correspond à un régime stable. Elle donne une statistique des vitesses et directions des courants par heure marée.

Les bilans de circulation sont définis à partir du calcul de l'intégrale de la projection des vecteurs vitesse, selon l'axe moyen des écoulements, (définis visuellement sur le scatterplot des vecteurs courant ou par régression linéaire), par le temps, et pour une surface unitaire de $1 \mathrm{~m}^{2}$. La convention adoptée est positif pour un flux entrant et négatif pour un flux sortant (du bassin lagonaire considéré).

où :

$$
Q=S * \sum_{t 0}^{t i} v i * \cos (\alpha i-\theta) * d t
$$




\section{V̀mes Journées Nationales Génie Côtier - Génie Civil \\ Toulon, 29 juin au $1^{\text {er }}$ juillet 2016}

$Q=$ volume d'eau ayant transité entre l'instant $t_{0}$ et l'instant $t_{i}$ en $\left(\mathrm{m}^{3}\right)$ pour $1 \mathrm{~m}^{2}$.

$S=$ surface $\left(\right.$ en $\left.^{2}\right)$ si supérieure à la surface unité $\left(1 \mathrm{~m}^{2}\right)$.

$v i=$ vitesse du courant $\mathrm{i}$ en $\mathrm{m} / \mathrm{s}$

$\theta=$ axe de la passe de flot $\left({ }^{\circ} \mathrm{N}\right)$.

$\alpha i=$ direction du vecteur courant $\mathrm{i}\left({ }^{\circ} \mathrm{N}\right)$.

$t i$ = temps écoulé depuis l'instant t0 (en s).

$d t=$ pas de temps.

\section{Conditions climatiques}

Le régime des vents est dominé par les alizés de sud-est qui soufflent entre $70 \%$ et $90 \%$ du temps (figure 1). Les vitesses de l'alizé sont le plus souvent modérées et dépassent rarement $10 \mathrm{~m} / \mathrm{s}$. Exceptionnellement, un régime de vents d'ouest à nord-ouest se développe, de $10 \%$ à $30 \%$ du temps. Il correspond souvent à du mauvais temps. En ce qui concerne les houles, une statistique réalisée sur 2005- 2010 à partir des données du site Iowaga-Ifremer, au point W176S13, montre que le Hs moyen s'établit à 1,82 m et la période moyenne, Tp, à 10,1 s. Les directions moyennes des houles sont un peu différentes de celle des vents (figure 2).

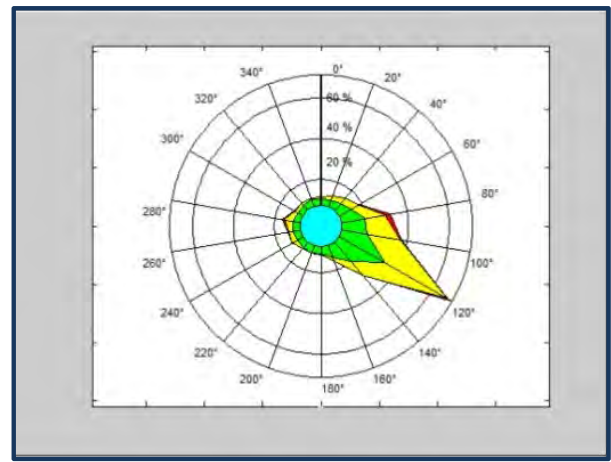

Figure 1. Rose des vents pendant la session de Te Fasi.

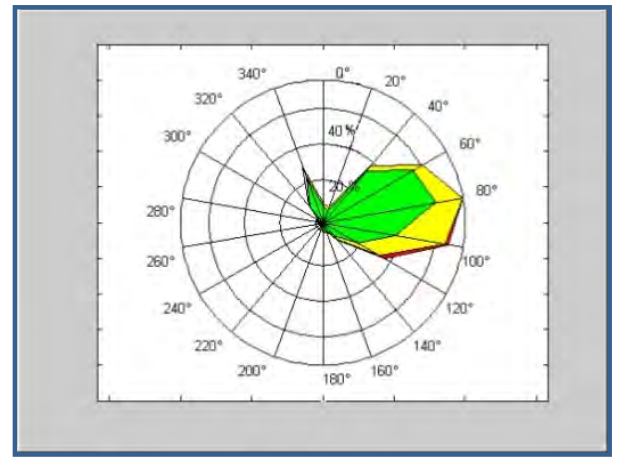

Figure 2. Rose des houles pendant la période 2005 - 2010.

\section{Résultats}

En l'absence de carte bathymétrique disponible, la figure 3 montre l'emplacement des stations sur la photo aérienne du site.

Les caractéristiques des courants mesurés en surface pour l'ensemble des stations des bassins nord sont rassemblées dans le tableau 1. Ce tableau donne les vitesses et la direction du courant de jusant ainsi que les bilans de circulation de la cellule de surface C1 pour une durée de 6 jours, soit une série de 288 mesures. 


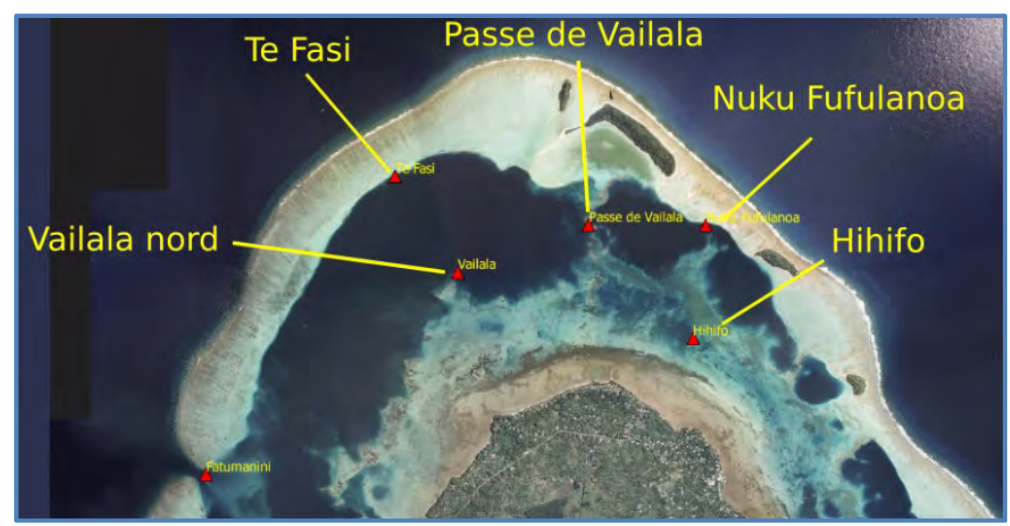

Figure 3. Position des stations du système lagonaire nord.

Tableau 1. Vitesses des courants et bilans de circulation en surface (C1) pour différentes stations du lagon nord.

\begin{tabular}{lrrrrr}
\hline Stations & Te fasi & Vailala $\boldsymbol{N}$ & Vailala P & Hihifo & Nuku F \\
Prof $(\mathrm{m})$ & 9,4 & 9,3 & 13,6 & 8,5 & 8,5 \\
$\operatorname{Vmax}(\mathrm{m} / \mathrm{s})$ & 0,61 & 0,87 & 0,61 & 0,57 & 0,62 \\
$\operatorname{Vmoy}(\mathrm{m} / \mathrm{s})$ & 0,20 & 0,42 & 0,20 & 0,26 & 0,23 \\
$\operatorname{Vol}\left(\mathrm{m3} / \mathrm{m}^{2}\right)$ & $-106233,3$ & $-83416,1$ & $-33037,1$ & $-158903,8$ & $-104272,9$ \\
Dir jusant & $275^{\circ}$ & $230^{\circ}$ & $230^{\circ}$ & $310^{\circ}$ & $320^{\circ}$ \\
\hline
\end{tabular}

\section{Stations de Hihifo et de Nuku Fufulanoa}

Ces stations de chenal interne montrent un régime courantologique caractéristique. Dans les conditions d'alizé normales, il se produit une évacuation des masses d'eau apportées par le déferlement des vagues sur le récif en direction de la fausse passe de Vailala. Cette direction d'écoulement est contrôlée par le tombant interne et la chenalisation. Exceptionnellement, lors des coups de vent d'ouest à nord-ouest, les eaux font le chemin inverse pour s'évacuer par-dessus la barrière récifale, le long du récif nord-est. En ce qui concerne les bilans de circulation à la station de Nuku Fufuloana (tableau 2), le maximum d'écoulement des eaux de surface est obtenu dans des conditions de marée de vives eaux et de houles modérées, (session S3), tandis que les écoulements de fond sont minimums. Le maximum d'écoulement pour l'ensemble de la tranche d'eau est obtenu dans des conditions de houles modérées et de marées de mortes-eaux (session S6).

Ceci s'explique par le fait que les couches de fond sont davantage influencées par la marée. En marées de vives eaux, le flot et le jusant s’équilibrent et le flot attenue les écoulements de fond. En marée de mortes eaux, les écoulements de jusants sont dominants sur toute la tranche d'eau et le flot n'atténue pas les écoulements de fond. L'asymétrie du cycle de marée est une indication du flux entrant par-dessus la barrière récifale 


\section{XIV èmes Journées Nationales Génie Côtier - Génie Civil \\ Toulon, 29 juin au $1^{\text {er }}$ juillet 2016}

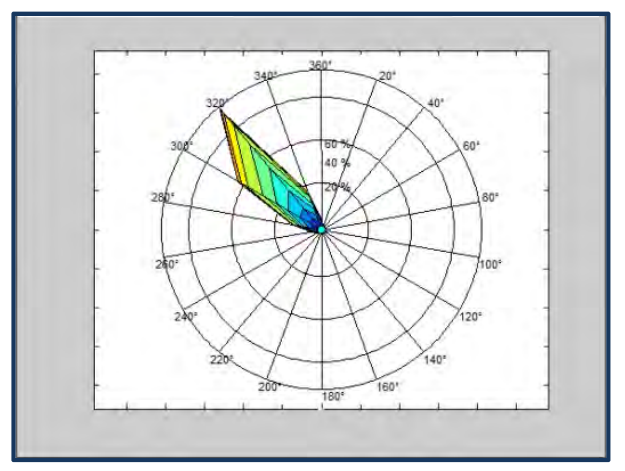

Figure 4. Rose des courants de la cellule de surface C1, à la station de Hihifo.

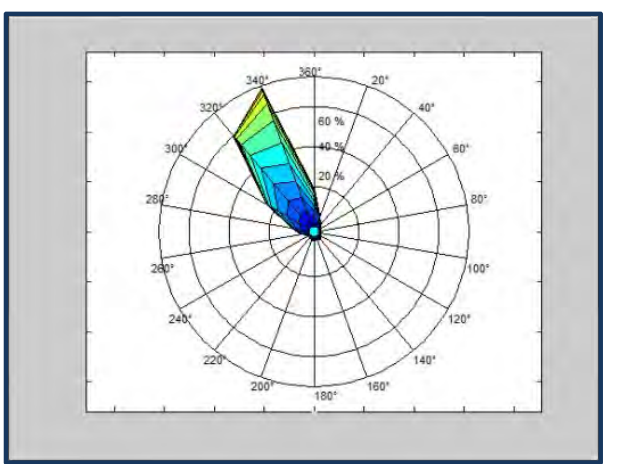

Figure 5. Rose des courants de la cellule de surface C1, à la station de Nuku Fufulanoa.

Tableau 2. Bilans de circulation cumulés $\left(\mathrm{m}^{3} / \mathrm{m}^{2}\right)$ par session, à la station de Nuku Fufulanoa, pour 240 mesures.

\begin{tabular}{llllllll}
\hline Sess & vent & marée & houle & C1 & C2 & C3 & Total \\
\hline S1 & SE & $V E$ & $S W$ & $-86894,1$ & $-59710,5$ & $-57182,9$ & $-203787,5$ \\
S2 & $N O$ & $M E$ & $L W$ & $-37704,8$ & $-43197,1$ & $-42826,1$ & $-123728,0$ \\
S3 & $S E$ & $V E$ & $L W$ & $-130036,8$ & $-31861,9$ & $-31413,8$ & $-193312,5$ \\
S4 & $S E$ & $V E$ & $S W$ & $-89440,2$ & $-47289,8$ & $-49264,4$ & $-185994,4$ \\
S6 & $S E$ & $M E$ & $L W$ & $-112615,4$ & $-68584,3$ & $-67871,6$ & $-249071,3$ \\
\hline \multicolumn{7}{c}{ Vent : SE : vent de sud-est, NO : vent d'ouest à nord-ouest } & \\
\multicolumn{6}{c}{ VE : marées de vives eaux, ME : marées de mortes eaux } \\
\multicolumn{6}{c}{ SW : forte houle - LW : mer calme } \\
\hline
\end{tabular}

\section{Station de Te Fasi}

Les observations de la station de Te Fasi montrent que, dans les conditions normales d'alizés de sud-est, les eaux de surface, en provenance du lagon est, via la passe de Vaialala, se déversent directement par-dessus la barrière récifale en direction du large. Pour les couches de fond, C3, C4, contrôlées par le tombant interne de la barrière récifale, le flux est redirigé en directions de la passe de Fatumanini située au sud-ouest (figure 6). C’est un régime quasi permanent qui n’est interrompu que pendant les coups d'Ouest (figure 8). Il se produit alors une inversion des courants. Les masses d'eau océaniques rentrent dans le lagon par-dessus la barrière récifale et s'évacuent ensuite, soit par les passes situées au sud-ouest (Fatumanini et Fuga Uvea), soit vers le lagon Est, par la fausse passe de Vailala).

L'étude des bilans de circulation pour la cellule de surface montre bien la différence entre le régime d'alizé (figure 7) et le régime de coup d’ouest (figure 8). On voit nettement l'inversion du bilan de circulation cumulé qui devient positif (sens de l'entrée d'eau dans le lagon) pendant une courte période (rectangle rouge). Ces épisodes sont généralement assez brefs (1 à 2 jours) et n'influent qu'assez peu sur le bilan de circulation global d'une session. 


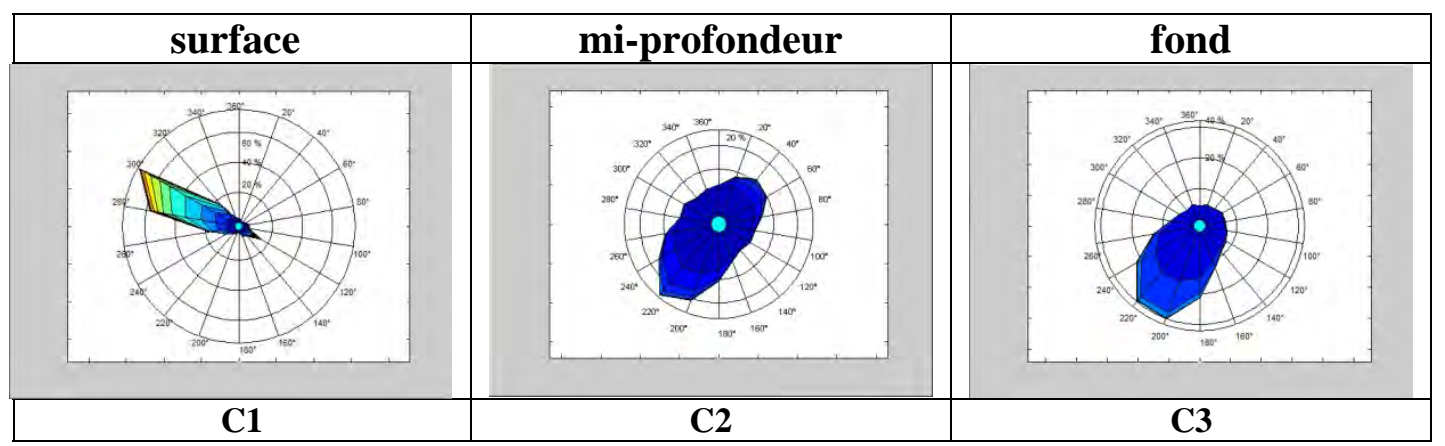

Figure 6. Rose des courants à la station de Te Fasi pour les cellules de surface de miprofondeur et de fond.

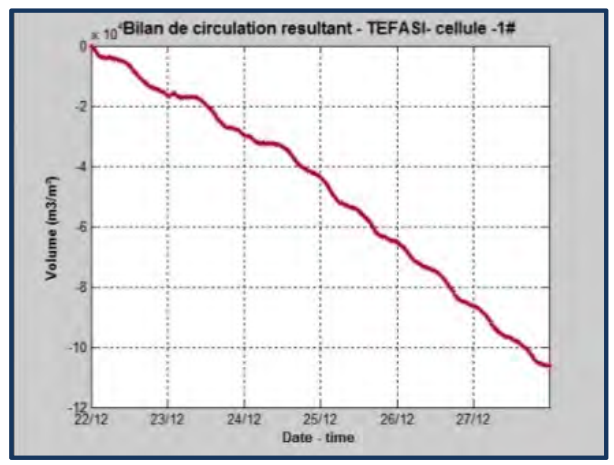

Figure 7. Bilan de circulation cumulé en régime normal d'alizés de sud-est.

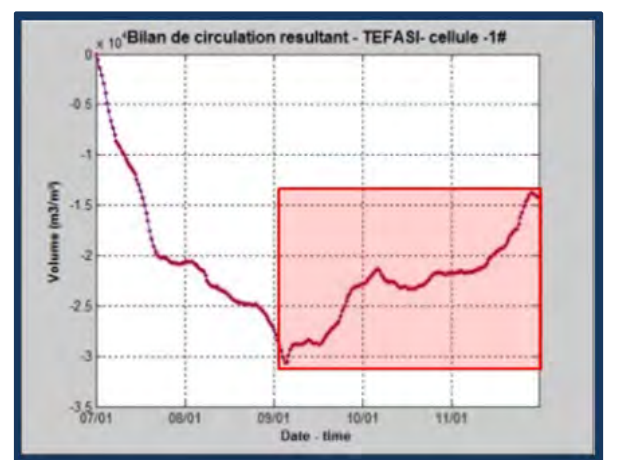

Figure 8. bilan de circulation cumulé lors d'un coup d'ouest (rectangle rouge).

\section{Circulation à deux couches}

L'analyse des résultats montrent le développement dans le lagon, d'une circulation à deux couches dont l'épaisseur est variable selon les stations et le régime courantologique.

- une couche superficielle très mobile, avec des courants rapides, influencée par les vents et les apports d'eau par les houles océaniques mais relativement peu influencée par la marée.

- une couche de fond, avec des courants moins rapides, plus réguliers, davantage influencés par la marée et contrôlées par la morphologie du fond (chenalisation, tombant récifal, etc.).

Un exemple de ce modèle de circulation à deux couches et donné par la station de Vailala Nord qui montre une circulation résiduelle opposée, en fonction de la profondeur (figure 9). Les cellules de surface (C1, C2) ont une circulation dominante dirigée vers le sud-ouest (vers la passe de Fatumanini) alors que la circulation de fond dominante (cellules C3, C4), est dirigée vers le nord-est (en direction de la passe de Vailala). Mais de nombreuses autres stations montrent des différences assez marquées entre la surface et le fond. 


\section{V̀mes Journées Nationales Génie Côtier - Génie Civil \\ Toulon, 29 juin au $1^{\text {er }}$ juillet 2016}

L'épaisseur de la couche de surface, variable de 3 à $5 \mathrm{~m}$, selon les stations, peut s'étendre plus en profondeur lorsque les conditions dynamiques deviennent plus sévères (fortes houles du large, cyclone).

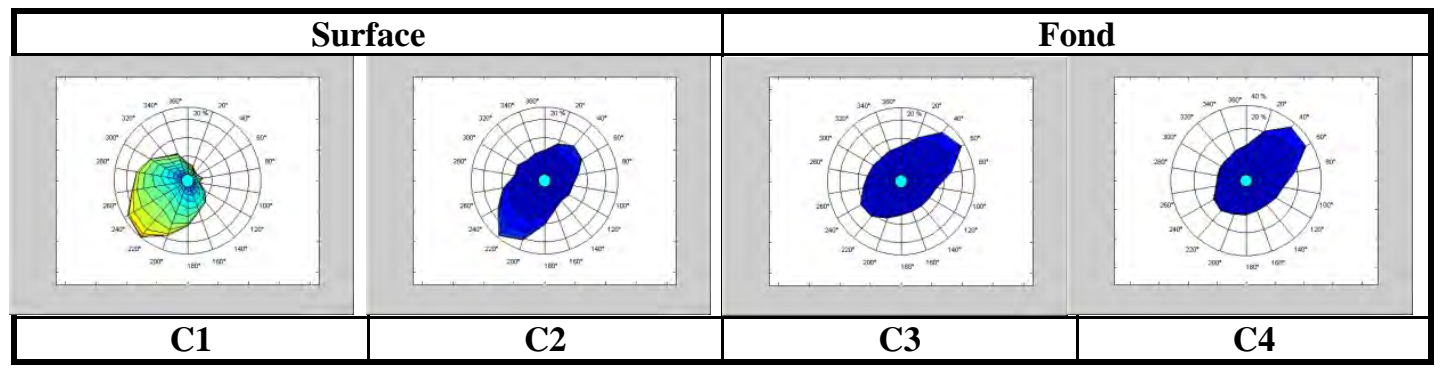

Figure 9. Roses des courants pour la station de pleine eau de Vailala Nord.

\section{Conclusions}

Le contrôle du modèle de circulation courantologique global par les conditions météorologiques se voit confirmé. Dans les conditions normales d'alizés de sud-est, les eaux du lagon nord-est remontent vers le Nord et se déversent dans le bassin nord-ouest par le biais des chenaux internes du lagon (chenaux de Hihifo, de Nuku Fufulanoa, et passe de Vailala). Elles traversent ensuite le lagon nord-ouest pour se déverser, soit directement par-dessus la barrière récifale, pour la couche de surface, soit par la passe de Fatumanini pour les eaux de fond (figure 10).

Lors des épisodes de vent d'ouest, les eaux font le chemin inverse pour rejoindre le lagon Est par la passe de Vailala (figure 11) et se déverser par-dessus la barrière récifale et par les fausses passes du récif (JUNKER, 2005).

Le modèle de circulation à deux couches est lui aussi détaillé. Il existe manifestement une couche de surface qui est soumise aux agents dynamiques comme le vent et les apports d'eaux océanique par-dessus la barrière récifale, qui estompent l'influence de la marée. Cette couche, dont l'épaisseur varie en fonction de la sévérité des conditions dynamiques, est assez mobile, et assure le drainage des eaux de surface.

Toutefois, la différenciation surface/fond n’a pas été observée dans les coupes des coupes T/S - profondeur (eaux en apparence homogènes).

Le calcul des bilans de circulation au cours de plusieurs cycles de marée et les harmoniques de courant permettent de déterminer les paramètres susceptibles de moduler la circulation ; ce sont principalement le type de marées (vives eaux et mortes eaux) et secondairement les entrées d'eau en période de fortes houles (Hs $\geq 3 \mathrm{~m}$ ).

\section{Remerciements}

Nos remerciements sont adressés au comité IFRECOR, au Service Territorial de l'Environnement de Wallis et Futuna et à l'Université de Nouvelle-Calédonie pour leur soutien dans ces travaux de recherche. 


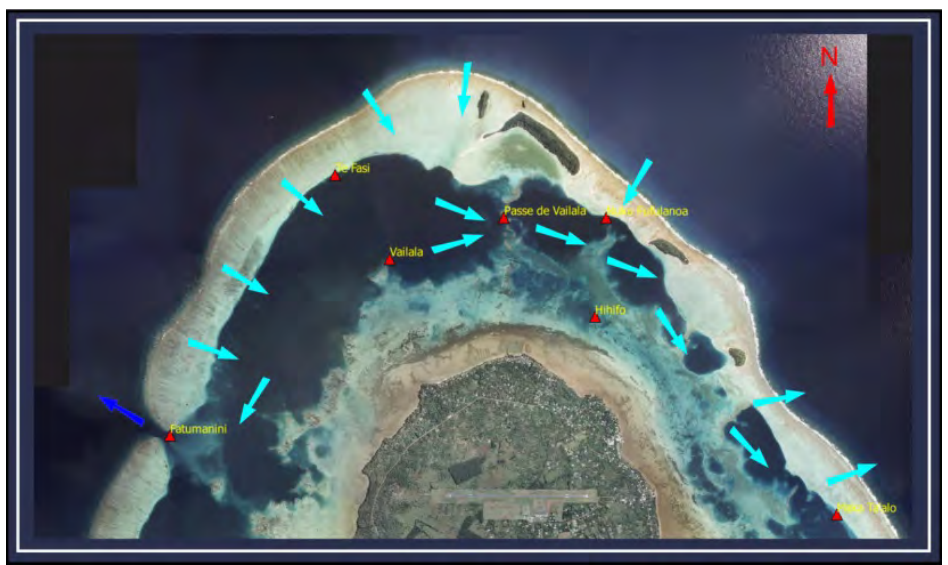

Figure 10. Schéma de circulation global dans le lagon nord dans les conditions normales d'alizés de sud-est.

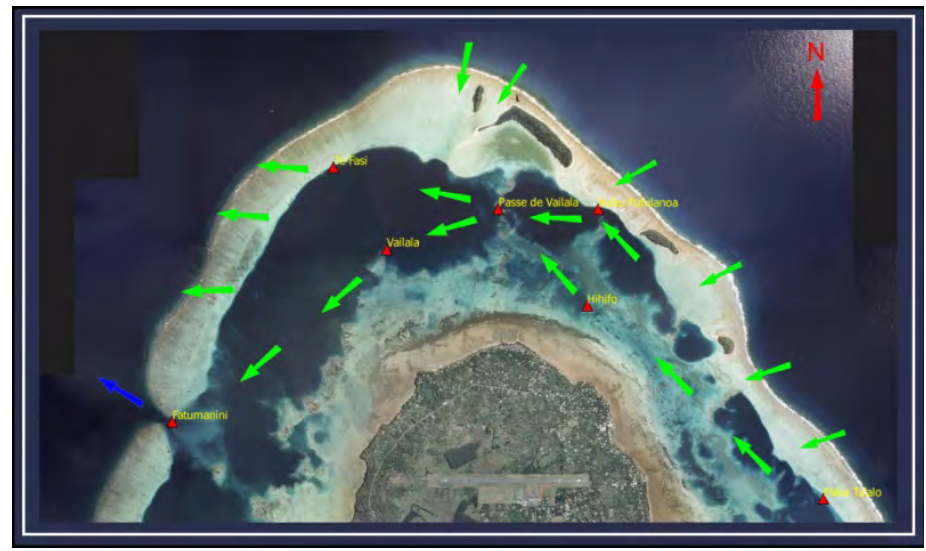

Figure 11. Schéma de circulation global dans le lagon nord, pendant une période de vent de nord-ouest.

\section{Références bibliographiques}

CHEVALIER C., SOUS D., DEVENON J.L., PAGANO M., ROUGIER G., BLANCHOT J. (2015). Impact of cross-reef water fluxes on lagoon dynamics: a simple parametrization for coral lagoon circulation model, with application to the Ouano Lagoon, New Caledonia. Ocean Dynamics, Vol. 65, pp 1509-1534. http://dx.doi.org/10.1007/s10236-015-0879-x

HOIBIAN T., ALLENBACH M. (2012). Caractérisation du bilan de circulation hydrologique du lagon de Wallis (Pacifique sud-ouest). Journée Nationales Génie Côtier Génie Civil Cherbourg, pp 933-940. http://dx.doi.org/10.5150/jngcgc.2012.102-H HOIBIAN T., LIUFAU E., ALLENBACH M., MALAU A. (2014). Développements récents dans la connaissance de l'océanologie du lagon de Wallis. Journée Nationales Génie Côtier Génie Civil, Dunkerque, pp 101-108. http://dx.doi.org/10.5150/jngcgc.2014.012 JUNCKER M. (2005). Approvisionnement en larves de poissons du lagon de Wallis (Pacifique Sud). Thèse de doctorat, Université de la Nouvelle-Calédonie. 